Published in final edited form as:

Curr Opin Obstet Gynecol. 2018 April ; 30(2): 116-122. doi:10.1097/GCO.0000000000000440.

\title{
Zika virus: A public health perspective
}

Nahida Chakhtoura, MD, Rohan Hazra, MD, and Catherine Y Spong, MD

Eunice Kennedy Shriver National Institute of Child Health and Human Development, National Institutes of Health; 31 Center Drive, 2A03 Bethesda MD 20892

\section{Abstract \\ Purpose of review-Zika virus' impact on pregnancy became widely known in 2015 with a dramatic increase in the number of babies born with microcephaly in Recife, Brazil. A mosquito borne virus resulting in congenital anomalies is unique, and Zika's ability to cause neurological defects on a large scale was a grim reminder of the Rubella epidemic in the 1950s. Over the past two years, studies have provided insight on how Zika virus (ZIKV) infects cells and causes disease, but much remains unknown about the long-term risks of Zika exposure on infant growth and development.}

Recent findings-The impact of ZIKV on pregnancy extends beyond microcephaly and may only first be identified in infancy. The virus has a long latency in semen and can be transmitted sexually. Transplacental passage occurs through infection of Hofbauer cells in the trophoblast. A major difficulty in management of ZIKV disease is that most infections are asymptomatic, and the diagnostic methods are not ideal, making both diagnosis and ascertainment of timing of infection problematic. Several different types of vaccines are in development. Large studies are ongoing to determine the risk and total spectrum of anomalies based on the timing of infection and other environmental exposures.

Summary-This review will summarize the epidemic, what we have learned, what we hope to learn, and current recommendations for care and management.

\section{Keywords}

Zika virus; pregnancy; microcephaly; child; anomaly

\section{Introduction}

Zika virus' impact on pregnancy emerged in 2015 with a dramatic increase in the number of babies born with microcephaly in Recife, Brazil (Figure). A virus resulting in congenital anomalies had not been seen to this degree since the Rubella epidemic in the 1950s, and its mosquito borne transmission was unique. Zika virus (ZIKV) is a single-stranded RNA arbovirus in the Flavivirus family, the same family that includes yellow fever, dengue, and West Nile. ZIKV is carried and can be transmitted by the Aedes aegypti mosquito. While

Contact information: Phone: 301496 1848, spongc@mail.nih.gov.

Conflicts of interest: none. 
other Flaviviruses can infect transplacentally, none have had the impact of ZIKV maternalfetal transmission. In addition, ZIKV can be transmitted sexually.

ZIKV was first identified in 1947 in Uganda's Zika forest, and the first human cases were detected in 1952. Although other outbreaks have occurred, most notably in the Yap islands in 2007 and French Polynesia in 2013, the link to fetal anomalies was not noted at the time. It was not until October 2015 that the association was recognized in Brazil with a dramatic rise in infants born with microcephaly. Soon after, on February 1, 2016, The World Health Organization declared ZIKV and its suspected link to birth defects an international public health emergency. Most ZIKV infections are mild; most of those infected (approximately $80 \%$ ) are asymptomatic. Symptoms, if they occur, typically last up to a week and include a maculopapular rash, fever, arthralgias, and non-purulent conjunctivitis. The viremia is short, and serological tests for antibodies cross-react with other flaviviruses, making it difficult to diagnose in areas where dengue virus is endemic. Polymerase chain reaction (PCR) testing is used to diagnose acute infection. This is only possible during the short, usually 5-day viremia period, which may be missed if the person is asymptomatic. These challenges highlight the difficulty of timing the exposure during pregnancy (1).

Treatment is directed at the symptoms. Despite intensive efforts, no antivirals or vaccines are currently available. The major focus has been on prevention, including reducing the mosquito population, limiting travel to endemic areas, and educating the public on methods to avoid mosquito bites and prevent sexual transmission. This review will summarize the epidemic, what we have learned, what we hope to learn, and current recommendations for care and management.

\section{ZIKV and other conditions}

ZIKV has a predilection for neural tissue (2). Infection can be severe even in non-pregnant people, causing Guillain-Barre syndrome (GBS), acute myelitis, meningoencephalitis, and acute disseminated encephalomyelitis (3). While other viral infections can result in GBS, $\mathrm{ZIKV}$-associated GBS is reported to be more severe, with a longer duration to improvement and incomplete resolution of the paralysis $(4,5)$.

\section{Modes of transmission and viral persistence}

In addition to being vector borne via the Aedes Aegypti mosquito, transmission can occur from mother to child, via sexual transmission, and via blood donation/transfusion or organ transplant. An evaluation of the incidence of ZIKV in non-pregnant, reproductive age men and women found $90 \%$ more cases of ZIKV per 100,000 in women than men, suggesting that sexual transmission is a major route of transmission (6). A similar study of dengue did not find a difference in this same age range, and dengue is not known to be transmitted sexually. Sexual transmission of Zika virus can occur after both symptomatic and asymptomatic infection.

Zika persists longest in semen. The virus has been reported to persist for 6 weeks with detection of the viral genome up to 180 days post infection. A recent study found median detection rates of 11 days in urine, 14 days in serum, and 42 days in semen and found that ninety-five percent of the participants cleared the virus by 40 days for serum, 37 days for 
urine, and 117 days for semen. This study also confirmed that the detection of viremia in pregnancy was longer than in non-pregnancy. (7).

\section{ZIKV and pregnancy}

Congenital zika syndrome (CZS) involves a spectrum of infant outcomes identified at birth (Table) $(8,9)$. The first reported studies focused only on symptomatic women and found $29 \%$ with fetal anomalies and $17 \%$ with microcephaly, cerebral atrophy, or intracranial calcifications. Importantly, they also reported that the risk was not limited to the first trimester; significant complications were observed with infection in the third trimester (10). A retrospective study focusing on the outbreak in French Polynesia suggested ZIKV in the first trimester resulted in a $1 \%$ rate of microcephaly, 50-fold higher than baseline (11). A more recent analysis of the U.S. Zika Pregnancy Registry from January to December 2016 reported a 15\% incidence of CZS when exposure was in the first trimester (12). Data from the same registry found an overall birth defects rate of $6 \%$; if the infection was in the first trimester, it rose to $11 \%$. Most of the defects were in the brain, including microcephaly (13).

CZS is associated with a wide variety of anomalies - especially neurologic and fetal growth abnormalities (Table) (9). These defects include central nervous system disruption, microcephaly, epileptic seizures, arthrogryposis, neuromotor delay that may include spasticity, exacerbated primitive reflexes, hyperexcitability, neurosensory deficits, and dysphagia. Early studies identified numerous ocular abnormalities in the children with microcephaly, including macular and perimacular lesions and optic nerve abnormalities (14). These studies further reinforced the hypothesis that ZIKV was the cause as perinatal viruses have previously been associated with these type of eye lesions. Neuroimaging studies highlight the range of fetal brain abnormalities, which include a collapse of the skull cap with overlapping sutures and prominent occipital bones, cortical development abnormalities, calcifications, ventriculomegaly, abnormalities of the corpus callosum, and brain stem abnormalities. Although intracerebral calcifications are common in perinatal viral infections, the pattern with ZIKV infection is unique, involving the cortical-subcortical junction, an area noted for vascular alterations (15). There is variability in the impact from infection in pregnancy (16), highlighting the need for well-designed studies of risk assessment.

Although viremia is noted to last only 3-5 days in symptomatic non-pregnant adults, studies of pregnant women and nonhuman primates have identified prolonged viremia in pregnancy. In one case report, viremia persisted for 10 weeks (17).

An asymptomatic ZIKV infection can also impact pregnancy outcome, with similar anomalies (18). Ongoing studies will evaluate the role of symptoms on the risk of infection in pregnancy. Although the long-term outcomes are not known, both motor and sensory abnormalities are anticipated $(19,20)$. It is likely that there will be a spectrum of outcomes including developmental delay, intellectual impairments, and mental and motor abnormalities. A concerning development has been the identification of acquired microcephaly in children exposed to ZIKV in utero and born with a normal head circumference and no apparent abnormalities. Follow-up studies of some children have diagnosed acquired microcephaly at 11-12 months (21). 


\section{Mechanism(s)}

Mouse models demonstrate that the placenta is uniquely affected by ZIKV (22). Replication of the virus in trophoblasts impairs placental function and may contribute to fetal growth restriction. Studies of infected human placental tissue in mid-gestation demonstrate focal proliferation of placental macrophages and enlarged hydropic chorionic villi (23). In the anchoring villus, replication occurs in proliferating cytotrophoblasts in proximal cell columns, in dividing Hofbauer cells in villus cores, and in invasive cytotrophoblasts. These suggest the ability to amplify infection in the basal decidua and chorionic villi as a method for transplacental passage (24).

Placental infection may impact the placenta itself and thus may cause fetal growth restriction or fetal death. If transplacental transmission occurs, the fetus itself may be infected, as has been noted with recovery of the virus from fetal brain tissue and eye abnormalities. The predilection for neural cell precursors with resultant apoptosis damages the developing fetal brain, resulting in microcephaly, blindness, and fetal loss. (25)

Clinical studies and animal models have revealed evidence that the Asian strain of ZIKV can directly target neural progenitor cells and cause microcephaly. Mouse models have demonstrated that ZIKV can replicate in the brain and infect neural progenitor cells, causing brain differentiation, apoptosis, and development to cease. $(26,27)$ It is suspected that functional adaptations in the virus, such as the prM protein, enables the virus to be more virulent. (28)

\section{Ongoing studies}

Numerous groups, both in industry and in government, are developing vaccines of various types, including DNA plasmid, mRNA, inactivated virus, self-amplifying RNA, liveattenuated, and viral vectors including vesicular stomatitis virus, adenovirus, and measles, though pregnant women have not been included in the early studies (29).

Basic work is ongoing using animal models, cell lines, and human tissues to further understanding the mechanisms of pathogenesis and identification of therapies (30). A limitation of the mouse model is that ZIKV does not replicate efficiently (31). Chen and colleagues identified that interferon-I can provide partial protection in this model. (32).

Several large Cohort studies are prospectively enrolling pregnant women in zika endemic regions to better characterize ZIKV infection in pregnancy and the short and long-term outcomes in infants. The Zika in Infants and Pregnancy Study (ZIP) is a collaboration between the National Institutes of Health and the Brazilian funding agency Fundacao Oswald Cruz (Fiocruz). It aims to enroll up to 10,000 pregnant women and follow them and their infants to evaluate the impact of ZIKV in pregnancy on maternal and child outcomes. This study will include an evaluation of the timing of infection during pregnancy, whether symptoms impact the outcome, and the role of other environmental factors. This study is ongoing at ten sites in six countries. The primary outcomes include congenital malformations and adverse fetal outcomes, including microcephaly, fetal demise, neonatal death, central nervous system malformations, hydrops, and ocular abnormalities. Numerous secondary outcomes are planned, including the rate of seroconversion, the impact of 
maternal symptoms on outcome, the impact of co-infections on outcome and disease severity, long-term clinical sequelae, persistence and shedding of Zika virus in tissue components, viral shedding in breast milk, and the incidence of acquisition of infection in infants after birth. Given that most Zika-infected women are asymptomatic, all infants will be followed for their outcomes. Thus, there will be three groups from the pregnancy cohort; those with symptomatic Zika virus infection, those with asymptomatic Zika virus infection, and those without Zika virus infections. During pregnancy, there will be a monthly exam, including physical exams and collection of biological samples; for each trimester there is an ultrasound exam and an environmental questionnaire. At delivery there is an infant examination and a collection of biologic samples, including maternal blood, cord blood, and placental samples. At six weeks postpartum there are maternal and infant examinations and a collection of biologic samples urine, blood, saliva, and maternal vaginal samples, and breast milk samples. After birth, examinations are performed on the infants at 3, 6, and 12 months, including physical and hearing assessments, ocular evaluation, and biologic sample collection. Imaging studies may also be performed. (33). Another ongoing study will evaluate the impact of co-infection with ZIKV and HIV. This study includes sites in the United States and Brazil. (34) Two additional large pregnancy cohort studies are ongoing, ZEN Columbia study (Zika in Pregnant Women and Children) in partnership with the CDC and INS (Instituto Nacional de Salud of Colombia), and European Zika Cohort studies Action/Alliance/Plan, multinational studies in Latin America and Asia.

The long-term impact of ZIKV on child outcomes is unclear (20). It is essential to monitor all children, given the identification of acquired microcephaly in infants who appeared unaffected at birth. Furthermore, it is likely that the spectrum of outcomes will be broad, and many may not manifest until school age.

\section{Management in pregnancy}

The first step in management during pregnancy includes education on the importance of prevention. The Centers for Disease Control and Prevention (CDC) operates numerous campaigns and efforts to educate the public on avoiding active areas of ZIKV transmission, including real-time updates on areas of concern (35). For those traveling to these areas, precautions to avoid mosquito bites include using insect repellent, wearing long sleeves, and using screens and mosquito netting. The importance of prevention has also been stressed for those returning from these areas to continue the precautions for two weeks. In addition, given the impact of sexual transmission, $\mathrm{CDC}$ has recommended avoidance of unprotected intercourse for 8 weeks after traveling for a woman and 6 months after traveling for a man, given the longer duration of virus in semen.

Numerous limitations are noted with Zika virus testing. Given that ZIKV RNA is only present for a limited time in body fluids, a negative result using nucleic acid testing does not rule out infection. Assessment of immunoglobulins requires careful timing, as a negative $\operatorname{IgM}$ may be due to assessment prior to mounting the IgM response or after the response has concluded. Recent work has found in some patients that the IgM response may persist in pregnant women, making it less useful in the timing of acute infection. Furthermore, the IgM cross-reacts with other flaviviruses, limiting its utility in areas where dengue virus is 
endemic. Finally, the IgM assays have noted both false-positive and false-negative results and they are currently being optimized by CDC. Due to these concerns, a recent revision of CDC guidelines includes offering RNA nucleic acid test (NAT) testing to asymptomatic pregnant women with ongoing possible ZIKV exposure. In addition, the IgM assay is no longer routinely recommended (36). Given the active work ongoing in this area, practitioners should monitor CDC recommendations for updated guidance (35)

For a pregnant woman with a positive Zika NAT test, careful anatomic survey and evaluation is recommended by ultrasound. Importantly, studies have demonstrated that there may be a significant lag between the timing of the infection and evidence of impact using ultrasound imaging (37). Furthermore, the sensitivity and specificity of ultrasound in the detection of CZS is not known. Nomograms for fetal head circumference have been developed. Pathologic microcephaly has been defined as a head circumference greater than or equal to 3 standard deviations below the mean (38). Serial ultrasound examinations may be beneficial for evaluating the development of any abnormalities and for monitoring fetal growth (39).

\section{Unexpected findings and Lessons learned}

Many aspects of the Zika story have been unexpected, perhaps most notably the ability for a mosquito borne virus to cause birth defects and for this virus to also be sexually transmitted. Zika's significant impact on all three trimesters of pregnancy is also unusual, as is the possibility of delayed impact in cases of acquired microcephaly diagnosed after birth. Although it is well appreciated that pregnancy offers a unique immune state, the prolonged viremia in pregnancy was not anticipated and may provide insights on the impact of the infection in pregnancy and the role of the placenta. The ability for "immune-protected" sites, including semen, brain, and lymphatic and optic tissues, to be reservoirs for prolonged periods has added to the complexity of counselling families. The affinity of ZIKV for neural tissue is unique and may provide insights for conditions that affect this system. Were it possible to remove the toxic aspects of the virus and replace them with a novel therapy, this might be an ideal vector for conditions that affect the nervous system. Difficulties in accurately diagnosing ZIKV infection have highlighted the critical need for rapid and advanced testing when facing a public health emergency, as counselling without definitive testing is exceedingly difficult.

\section{Conclusion}

ZIKV in pregnancy has highlighted the critical limitations faced by pregnant women and families during a public health emergency. Although it is not uncommon to have limited therapies in pregnancy, the lack of diagnostics and other information hinders the ability to counsel families. Furthermore, even with rapid mobilization for vaccine development, pregnant women are not included in the target population to benefit from these vaccines (40). It is critical for future public health emergencies to embrace the potential impact on pregnant women, children, and their families, and to include them in research, development, and planning processes. 


\section{Acknowledgments}

We would like to thank Mr. Paul Williams for his editorial assistance with this article.

Financial support and sponsorship: none.

\section{References}

1*. de Vasconcelos ZFM, Azevedo RC, Thompson N, Gomes L, et al. Challenges for molecular and serological ZIKV infection confirmation. Childs Nerv Syst. 2017 Nov 6. [Epub ahead of print]. This article outlines the challenges with diagnostics to identify zika virus infection, including the cross-reactivity with other flaviviruses. doi: 10.1007/s00381-017-3641-5

2*. Medina MT, Medina-Montoya M. New spectrum of the neurologic consequences of Zika. J Neurol Sci. 2017 Nov 1. pii: S0022-510X(17)34426-X. [Epub ahead of print]. This editorial outlined the role of zika as a new neuropatholical agent, adding the new report of childhood arterial ischemic stroke. doi: 10.1016/j.jns.2017.10.046

3. Méndez N, Oviedo-Pastrana M, Mattar S, Caicedo-Castro I, Arrieta G. Zika virus disease, microcephaly and Guillain-Barré syndrome in Colombia: epidemiological situation during 21 months of the Zika virus outbreak, 2015-2017. Arch Public Health. 2017 Nov 2.75:65. eCollection 2017. doi: 10.1186/s13690-017-0233-5 [PubMed: 29118981]

4. Sebastián UU, Ricardo AVA, Alvarez BC, et al. Zika virus-induced neurological critical illness in Latin America: Severe Guillain-Barre Syndrome and encephalitis. J Crit Care. 2017 Aug 9.42:275281. DOI: 10.1016/j.jcrc.2017.07.038 [PubMed: 28806562]

5. da Silva IRF, Frontera JA, Bispo de Filippis AM, et al. Neurologic Complications Associated With the Zika Virus in Brazilian Adults. JAMA Neurol. 2017 Oct 1; 74(10):1190-1198. DOI: 10.1001/ jamaneurol.2017.1703 [PubMed: 28806453]

6*. Coelho FC, Durnovni B, Saraceni V, et al. Higher incidence of zika in adult women than adult men in Rio de Janeiro suggests a significant contribution of sexual transmission from men to women. Int J Infect Dis. 2016; 51:128-132. This study highlighted the difference in incidence of zika virus infection between non-pregnant women and men in the reproductive age range (15-60) demonstrating the role of sexual transmission. [PubMed: 27664930]

7. Paz-Bailey G, et al. Zika virus persistence in body fluids, final report in body fluids- Final report. ASTMH. 2017 Abstract LB-5162.

8. Moore CA, Staples JE, Dobyns WB, et al. Characterizing the Pattern of Anomalies in Congenital Zika Syndrome for Pediatric Clinicians. JAMA Pediatr. 2017 Mar 1; 171(3):288-295. DOI:

10.1001/jamapediatrics.2016.3982 [PubMed: 27812690]

9*. Saad T, Pennae Costa AA, de Góes FV, de Freitas M, de Almeida JV, de Santa Ignêz LJ, Amancio AP, Alvim RJ, Antunes Kramberger LA. Neurological manifestations of congenital Zika virus infection. Childs Nerv Syst. 2017 Nov 10. [Epub ahead of print]. This article reports congenital zika syndrome and highlights the importance to followup children exposed to zika, even those who appear normal at birth. doi: 10.1007/s00381-017-3634-4

10**. Brasil P, Pereira JP Jr, Moreira ME, et al. Zika Virus Infection in Pregnant Women in Rio de Janeiro. N Engl J Med. 2016 Dec 15; 375(24):2321-2334. Epub 2016 Mar 4. This was one of the first reports detailing the anomalies associated with zika infection during pregnancy. In this cohort, $46 \%$ of the pregnancies had adverse outcomes regardless of the trimester of infection. [PubMed: 26943629]

11 * Cauchemez S, Besnard M, Bompard P, et al. Association between zika virus and microcephaly in French Polynesia 2013-2015: A retrospective study. Lancet. 2016; 387:2125-32. This study went back to look at the rate of microcephaly from the outbreak in French Polynesia suggested zika infection in the first trimester resulted in a 1\% rate of microcephaly. [PubMed: 26993883]

12. Reynolds MR, Jones AM, Petersen EE, et al. Vital Signs: Update on Zika Virus-Associated Birth Defects and Evaluation of All U.S. Infants with Congenital Zika Virus Exposure - U.S. Zika Pregnancy Registry, 2016. MMWR Morb Mortal Wkly Rep. 2017 Apr 7; 66(13):366-373. DOI: 10.15585/mmwr.mm6613e1 [PubMed: 28384133] 
13*. Honein MA, Dawson AL, Petersen EE, et al. Birth Defects Among Fetuses and Infants of US Women With Evidence of Possible Zika Virus Infection During Pregnancy. JAMA. 2017 Jan 3; 317(1):59-68. This article provided the rates of birth defects from the US based registry of zika infection in pregnancy. There were 21 infants with birth defects of 395 live births and 5 fetuses with anomalies of 47 pregnancy losses. Birth defects occurred equally (6\% each) in women with or without symptoms. DOI: 10.1001/jama.2016.19006 [PubMed: 27960197]

14. de Paula Freitas B, de Oliveira Dias JR, Prazeres J, et al. Ocular Findings in Infants With Microcephaly Associated With Presumed Zika Virus Congenital Infection in Salvador, Brazil. JAMA Ophthalmol. 2016 Feb 9. [Epub ahead of print]. doi: 10.1001/jamaophthalmol.2016.0267

15*. Ribeiro BNF, Muniz BC, Gasparetto EL, Ventura N, Marchiori E. Congenital Zika syndrome and neuroimaging findings: what do we know so far? Radiol Bras. 2017 Sep-Oct;50(5):314-322. Review. This review article summarizes the computed tomography and magnetic resonance imaging work of abnormalities seen with congenital zika syndrome. The review both describes the findings and illustrates them with clear images. DOI: 10.1590/0100-3984.2017.0098 [PubMed: 29085165]

16*. Nogueira ML, Rocha Nery, Júnior NR, Estofolete CF, et al. Adverse birth outcomes associated with Zika virus exposure during pregnancy in São José do Rio Preto, Brazil. Clin Microbiol Infect. 2017 Nov 10. This retrospective study evaluated the impact of zika virus in Colombia including pregnancy outcomes and Guillain-Barre syndrome.

17. Driggers RW, Ho CY, Korhonen EM. Zika Virus Infection with Prolonged Maternal Viremia and Fetal Brain Abnormalities. N Engl J Med. 2016 Jun 2; 374(22):2142-51. Epub 2016 Mar 30. DOI: 10.1056/NEJMoa1601824 [PubMed: 27028667]

18. Ventura CV, Maia M, Dias N, et al. Zika: neurological and ocular findings in infant without microcephaly. Lancet. 2016 Jun 18.387(10037):2502. Epub 2016 Jun 7. doi: 10.1016/ S0140-6736(16)30776-0 [PubMed: 27287830]

19*. da Silva Pone MV, Moura Pone S, Araujo Zin A, Barros Mendes PH, et al. Zika virus infection in children: epidemiology and clinical manifestations. Childs Nerv Syst. 2017 Nov 6. [Epub ahead of print] This review of congential zika syndrome highlights what is and is not known, concluding that the full spsectrum is not yet known. doi: 10.1007/s00381-017-3635-3

20**. Kapogiannis BG, Chakhtoura N, Hazra R, Spong CY. Bridging Knowledge Gaps to Understand How Zika Virus Exposure and Infection Affect Child Development. JAMA Pediatr. 2017 May 1; 171(5):478-485. This article summarizes a research agenda to improve the evaluation, monitoring, and management of neonates, infants, or children affected by ZIKV and its complications. The agenda also aims to optimally address the prospective effect of ZIKV exposure on the developing child. The manuscript summarizes concepts from a NIH workshop held September 2016. DOI: 10.1001/jamapediatrics.2017.0002 [PubMed: 28241263]

$21^{* *}$. van der Linden V, Pessoa A, Dobyns W, et al. Description of 13 Infants Born During October 2015-January 2016 With Congenital Zika Virus Infection Without Microcephaly at Birth - Brazil. MMWR Morb Mortal Wkly Rep. 2016 Dec 2; 65(47):1343-1348. This article was the first to describe deceleration of infant head circumference, the identification of acquired microcephaly. DOI: 10.15585/mmwr.mm6547e2 [PubMed: 27906905]

22. Miner JJ, Cao B, Govero J, Smith AM, et al. Zika Virus Infection during Pregnancy in Mice Causes Placental Damage and Fetal Demise. Cell. 2016; 165:1081-1091. [PubMed: 27180225]

23. Rosenberg AS, Yu W, Hill DA, et al. Placental pathology of zika virus: viral infection of the placenta induces villous stromal macrophage (Hofbauer cell) Proliferation and Hyperplasia. Arch Pathol Lab Med. 2017; 141(11):43-8. [PubMed: 27681334]

24**. Tabata T, Petitt M, Puerta-Guardo H, Michlmayr D, Harris E, Pereira L. Zika Virus Replicates in Proliferating Cells in Explants from First-trimester Human Placentas, Potential Sites for Dissemination of Infection. J Infect Dis. 2017 Nov 2. [Epub ahead of print] This basic science study evaluated primary cells from human placentals, evaluating viral replication in the villi and basal decidua. They found that zika replicated at consistent sites in the anchoring villus. doi: 10.1093/infdis/jix552

25. Platt DJ, Miner JJ. Consequences of congenital Zika virus infection. Curr Opin Virol. 2017 Oct 25.27:1-7. [Epub ahead of print] Review. PMID:29080429 This review highlights the impact of congenital zika virus infection. DOI: 10.1016/j.coviro.2017.09.005 [PubMed: 29080429] 
26. Tang H, Hammack C, Ogden SC, et al. Zika Virus Infects Human Cortical Neural Progenitors and Attenuates Their Growth. Cell Stem Cell. 2016 May 5; 18(5):587-90. Epub 2016 Mar 4. DOI: 10.1016/j.stem.2016.02.016 [PubMed: 26952870]

27. Li Y, Muffat J, Omer A, et al. Induction of Expansion and Folding in Human Cerebral Organoids. Cell Stem Cell. 2017 Mar 2; 20(3):385-396.e3. Epub 2016 Dec 29. DOI: 10.1016/j.stem. 2016.11.017 [PubMed: 28041895]

$28 * *$. Yuan L, Huang XY, Liu ZY, Zhang F, et al. A single mutation in the prM protein of Zika virus contributes to fetal microcephaly. Science. 2017 Nov 17; 358(6365):933-936. Epub 2017 Sep 28 This article identified that a single serine-to-asparagine substitution increased infectivity in both human and mouse neural progenitor cells with higher rates of microcephaly and mortality. This substitution arose prior to the outbreak in French Polynesia in 2013 and may explain the higher rates of microcephaly in recent epidemics. DOI: 10.1126/science.aam7120 [PubMed: 28971967]

29. Plotkin SA, Graham BS. Zika Virus. This chapter outlines the development of the zika virus vaccine.

30*. Krause KK, Azouz F, Shin OS, Kumar M. Understanding the Pathogenesis of Zika Virus Infection Using Animal Models. Immune Netw. 2017 Oct; 17(5):287-297. Epub 2017 Oct 19. Review. This review article outlines the small and large animal models used in zika virus research. DOI: 10.4110/in.2017.17.5.287 [PubMed: 29093650]

31*. Lazear HM, Govero J, Smith AM, Platt DJ, Fernandez E, Miner JJ, Diamond MS. A Mouse Model of Zika Virus Pathogenesis. Cell Host Microbe. 2016 May 11; 19(5):720-30. Epub 2016 Apr 5. This study evaluated zika infection in mouse models including those lacking components of the antiviral response. DOI: 10.1016/j.chom.2016.03.010 [PubMed: 27066744]

32*. Chen J, Liang Y, Yi P, Xu L, et al. Outcomes of Congenital Zika Disease Depend on Timing of Infection and Maternal-Fetal Interferon Action. Cell Rep. 2017 Nov 7; 21(6):1588-1599. This study using a mouse model demonstrated that fetus derived interferon- 1 is able to provide some protection against zika virus infection, outlining the role of interferon- 1 in the placenta as a potential therapeutic. DOI: 10.1016/j.celrep.2017.10.059 [PubMed: 29117563]

33. Zika in Infants and Pregnancy (ZIP) in Clinical Trials.gov. https://www.clinicaltrials.gov/ct2/show/ NCT02856984?term=nih+zip\&rank=1; accessed 12/5/17

34. Prospective Cohort Study of HIV and Zika in Infants and Pregnancy (HIV ZIP) in clinical trials.gov. https://www.clinicaltrials.gov/ct2/show/NCT03263195?term=zip+hiv\&rank=1; accessed $12 / 5 / 17$

35. https://www.cdc.gov/zika/pregnancy/index.html and www.cdc.gov; accessed 12/5/17

36. Adebanjo T, Godfred-Cato S, Viens L, et al. Udate: Interim Guidance for the Diagnosis, Evaluation and Management of Infants with Possible Congenital Zika Virus Infection-United States, October 2017. MMWR. Oct 20; 2017 66(41):1089-1099. [PubMed: 29049277]

37. Parra-Saavedra M, Reefhuis J, Piraquive JP, et al. Serial Head and Brain Imaging of 17 Fetuses With Confirmed Zika Virus Infection in Colombia, South America. Obstet Gynecol. 2017 Jul; 130(1):207-212. DOI: 10.1097/AOG.0000000000002105 [PubMed: 28594771]

38. Society for Maternal-Fetal Medicine (SMFM) Publications Committee. Ultrasound screening for fetal microcephaly following Zika virus exposure. Am J Obstet Gynecol. 2016 Jun; 214(6):B2-4. Epub 2016 Feb 19. No abstract. DOI: 10.1016/j.ajog.2016.02.043 [PubMed: 26901275]

39. Practice Advisory Interim Guidance for Care of Obstetric Patients During a Zika Virus Outbreak. https://www.acog.org/About-ACOG/News-Room/Practice-Advisories/Practice-Advisory-InterimGuidance-for-Care-of-Obstetric-Patients-During-a-Zika-Virus-Outbreak\#top accessed 12/5/2017

40**. Ethics Working Group on ZIKV Research and Pregnancy. Ethics, pregnancy, and ZIKV vaccine research \& development. Vaccine. 2017 Dec 14; 35(49 Pt B):6819-6822. Epub 2017 Oct 19. This document outlines the importance of evaluating the zika virus vaccine in reproductive age and pregnant women, a main target for the vaccine. DOI: 10.1016/j.vaccine.2017.09.065 [PubMed: 29056425] 


\section{Key points}

- $\quad$ Zika virus infection in pregnancy is more than microcephaly. Numerous systems are affected, and the implications of exposure may not be evident at birth.

- $\quad$ Acquired microcephaly has been identified in children at 11-12 months who were born with a normal-sized head and no apparent anomalies. It is critical to follow all children, not just those with visible anomalies at birth.

- $\quad$ Transmission can occur via a mosquito bite, blood transfusion, vertically from mother to child, or via sexual transmission.

- $\quad$ There are no antivirals or vaccines available, so prevention messages, including recommendations on avoiding of mosquito bites and education on sexual ZIKV transmission, is currently the best strategy to combat the epidemic. 


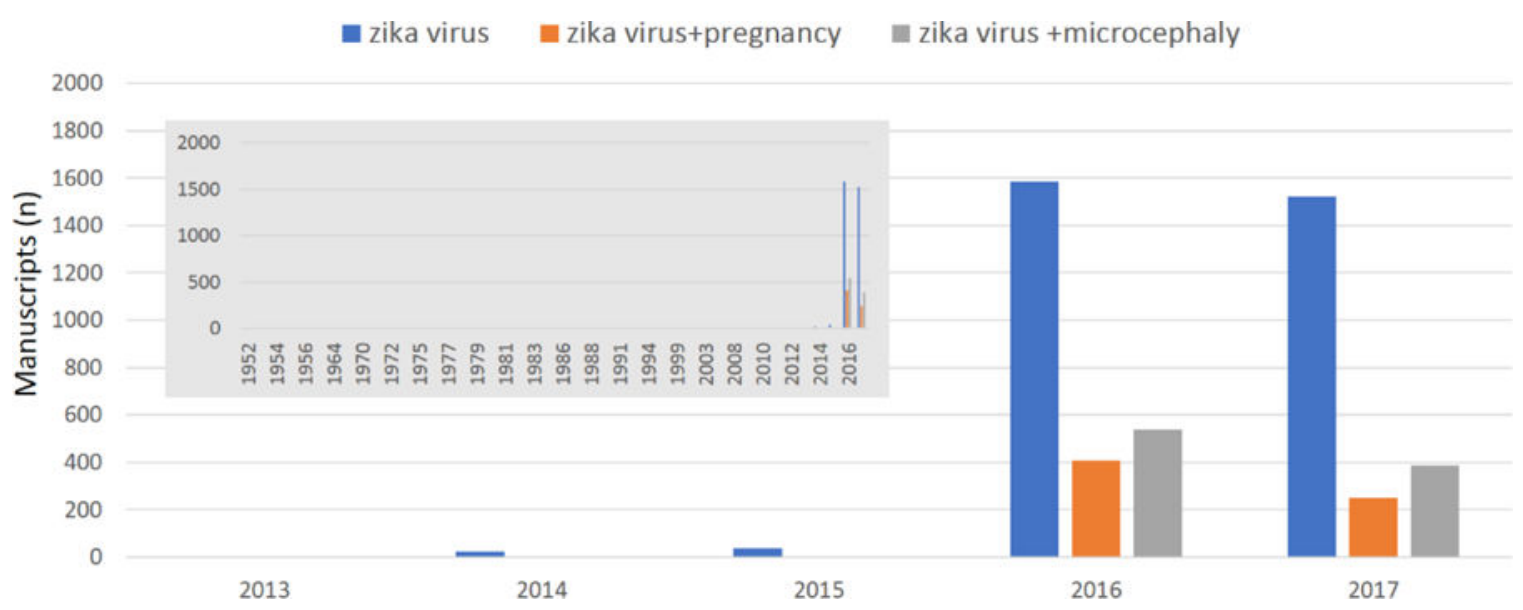

Figure 1.

Despite identification of zika virus in the 1940s, publications in PubMed did not explode until 2016. A search of PubMed (performed on 11/17/2017) on "zika virus" found single digit publications dating back to 1952 (two in 1952), with the number increasing to double digits in 2014 ( $\mathrm{n=23}$ ) and then over 1500 in 2016 and 2017 (insert graph upper left). A search of zika virus and pregnancy pulled one article in 2014 and 2015 with 408 in 2016 and 250 in 2017. Zika virus and microcephaly revealed 4 articles in 2015, 541 in 2016, and 386 in 2017. 


\section{Table 1}

Zika associate pregnancy outcomes extend far beyond the initial association with microcephaly. Those reported in the literature are summarized in this table

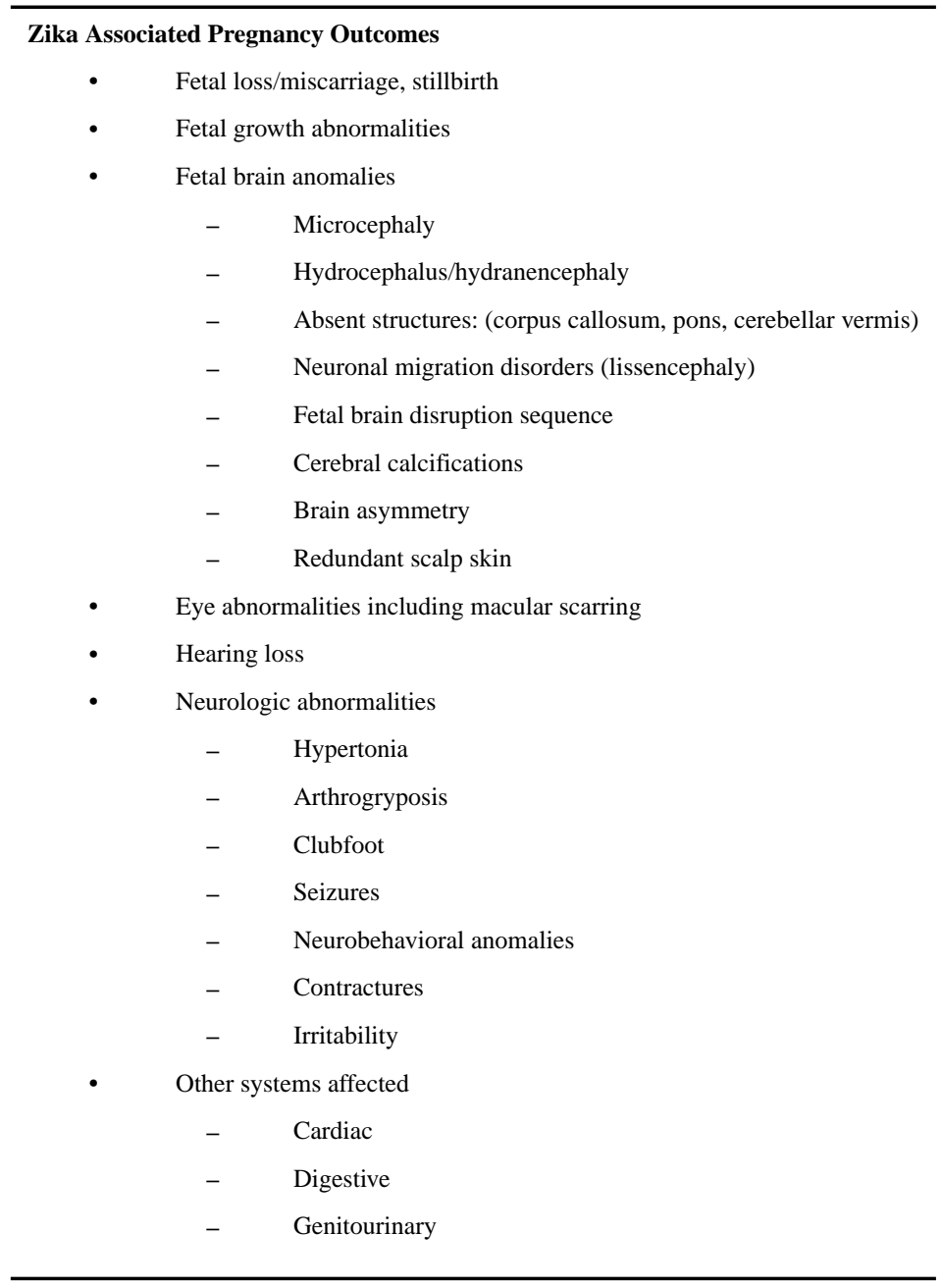

